\title{
See a Black Hole on a Shoestring
}

\author{
Jeremy S. Heyl \\ Department of Physics and Astronomy, University of British Columbia \\ 6224 Agricultural Road, Vancouver, British Columbia, Canada, V6T 1Z1; Canada Research Chair
}

(Dated: July 7, 2021)

\begin{abstract}
The modes of vibration of hanging and partially supported strings provide useful analogies to scalar fields travelling through spacetimes that admit conformally flat spatial sections. This wide class of spacetimes includes static, spherically symmetric spacetimes. The modes of a spacetime where the scale factor depends as a power-law on one of the coordinates provide a useful starting point and yield a new classification of these spacetimes on the basis of the shape of the string analogue. The family of corresponding strings follow a family of curves related to the cycloid, denoted here as hypercycloids (for reasons that will become apparent). Like the spacetimes that they emulate these strings exhibit horizons, typically at their bottommost points where the string tension vanishes; therefore, hanging strings may provide a new avenue for the exploration of the quantum mechanics of horizons.
\end{abstract}

\section{INTRODUCTION}

The rules governing the interaction of black holes in relativity bears a remarkable similarity to the laws of thermodynamics established with laboratory experiments 1, 2]. Over the past several years there has been great excitement about bringing the resemblance full circle with the description and realization of laboratory analogues to the structure of curved spacetimes [3, 4]. Studying these analogues has given new insight on how the properties of black hole horizons probe the underlying theory of quantum gravity [5]. These laboratory systems have generally involved surface or pressure oscillations of superfluids [4] and Bose-Einstein condensates [6] , and more recently microwaves in specially designed waveguides [7].

Although there has been substantial work on the dynamics of acoustic oscillations near vortexes, much of the effort to understand acoustical analogues to spacetimes has focused on $(1+1)$-dimensional systems $[8$, e.g.] that are either static or stationary. By dimensional reduction (focusing only on the radial modes), a general spherically symmetric spacetime in $(3+1)$-dimensional can be represented by a $(1+1)$-dimensional analogue [9]. This letter examines possibly the simplest realization of a $(1+1)$-dimensional analogue to spacetime, transverse oscillations travelling along a string - this is a layperson's string like a guitar or violin string rather than a fundamental or cosmic string. By varying the tension along the string, one can tune the properties of the analogue spacetime and even produce acoustic horizons along the string.

\section{WAVES ON A STRING}

The action for oscillations on a string with varying tension and mass density is given by [10, 11, p. 597, 598]

$$
S=\frac{1}{2} \int d s d t\left[\mu(s)\left(\frac{\partial \phi}{\partial t}\right)^{2}-\lambda(s)\left(\frac{\partial \phi}{\partial s}\right)^{2}\right]
$$

$$
=\frac{1}{2} \int d s d t \mu(s)\left[\left(\frac{\partial \phi}{\partial t}\right)^{2}-c_{s}^{2}(s)\left(\frac{\partial \phi}{\partial s}\right)^{2}\right]
$$

where $\mu$ is the mass per unit length of the string, $c_{s}^{2}=$ $\lambda / \mu$ and $\phi$ is the displacement of the string in one direction, and the tension $\lambda(s)$ is not affected by the oscillations. Let's define $c_{s} d \chi=d s$ and change variables in the integral to get

$$
S=\frac{1}{2} \int d \chi d t a^{2}(\chi)\left[\left(\frac{\partial \phi}{\partial t}\right)^{2}-\left(\frac{\partial \phi}{\partial \chi}\right)^{2}\right]
$$

where $a^{2}(s)=c_{s}(s) \mu(s)$.

This action for the acoustic modes along a string is the same as the action for a scalar field in a conformally flat spacetime. $\S \amalg$ will extend this result to spacetimes with conformally flat spatial sections.

Let's make the substitution $u=a \phi$ to try to absorb the factor of $a^{2}(\chi)$ into the fields.

$$
\begin{aligned}
S & =\frac{1}{2} \int d \chi d t\left[\left(\frac{\partial u}{\partial t}\right)^{2}-\left(a \frac{\partial(u / a(\chi))}{\partial \chi}\right)^{2}\right] \\
& =\frac{1}{2} \int d \chi d t\left[\left(\frac{\partial u}{\partial t}\right)^{2}-\left(\frac{\partial u}{\partial \chi}\right)^{2}-m_{\mathrm{eff}}^{2} u^{2}\right]
\end{aligned}
$$

where to get the final result we have dropped a term in the integrand equal to a total derivative with respect to the coordinate $\chi$, specifically we take

$$
\frac{1}{2} \int d \chi d t \frac{\mathrm{d}}{\mathrm{d} \chi}\left(\frac{u^{2}}{a} \frac{\partial a}{\partial \chi}\right)=0
$$

We find that the oscillations of the string acquire an effective mass of

$$
m_{\mathrm{eff}}^{2}=\frac{1}{a} \frac{\partial^{2} a}{\partial \chi^{2}}
$$




\section{A. Quantizing the String}

The first step to determining the quantum mechanics of the string is to obtain the Hamiltonian,

$$
H=\frac{1}{2} \int d \chi\left[\left(\frac{\partial u}{\partial t}\right)^{2}+\left(\frac{\partial u}{\partial \chi}\right)^{2}+m_{\mathrm{eff}}^{2} u^{2}\right]
$$

To look at the quantum mechanics of this Hamiltonian we must define the field operator $\hat{u}(\chi, t)$,

$$
\hat{u}(\chi, t)=\int d \omega\left[g(\omega, \chi) e^{-i \omega t} \hat{a}_{\omega}+g^{*}(\omega, \chi) e^{i \omega t} \hat{a}_{\omega}^{\dagger}\right]
$$

where $\hat{a}_{\omega}^{\dagger}$ and $\hat{a}_{\omega}$ are creation and annihilation operators that satisfy the following standard commutator relations

$$
\left[\hat{a}_{\omega}^{\dagger}, \hat{a}_{\omega^{\prime}}^{\dagger}\right]=\left[\hat{a}_{\omega}, \hat{a}_{\omega^{\prime}}\right]=0,\left[\hat{a}_{\omega}, \hat{a}_{\omega^{\prime}}^{\dagger}\right]=\delta\left(\omega-\omega^{\prime}\right) .
$$

Because the Hamiltonian does not explicitly depend on time, we are free to choose that the field operator depend harmonically on time.

If we assume that the function $g(\omega, \chi)$ solves the following differential equation

$$
\frac{\partial^{2} g}{\partial \chi^{2}}+\left(\omega^{2}-m_{\mathrm{eff}}^{2}\right) g=0
$$

then the Hamiltonian operator takes the simple form

$$
\hat{H}=\frac{1}{2} \int d \chi d \omega \omega\left(\hat{a}_{\omega} \hat{a}_{\omega}^{\dagger}+\hat{a}_{\omega}^{\dagger} \hat{a}_{\omega}\right)
$$

\section{B. Linear Tension}

Let's calculate $a(s)=\sqrt{c_{s}(s) \mu(s)}$ if the string is hanging vertically with one end of the string loose at $s=0$ and $\mu(s)=b s^{\alpha}$. We have

$$
\lambda(s)=\int_{0}^{s} g \mu\left(s^{\prime}\right) d s^{\prime}=\int_{0}^{s} g b\left(s^{\prime}\right)^{\alpha} d s^{\prime}=\frac{b s^{\alpha+1}}{\alpha+1},
$$

so

$$
c_{s}=\sqrt{\frac{g s}{\alpha+1}}, \chi=2 \sqrt{\frac{s}{g}(\alpha+1)} .
$$

for $\alpha>-1$. Putting things together we have

$$
a(\chi)=b^{1 / 2}\left(\frac{\chi}{2}\right)^{\alpha+1 / 2}\left(\frac{g}{\alpha+1}\right)^{(\alpha+1) / 2}
$$

and

$$
m_{\mathrm{eff}}^{2}=\frac{1}{\chi^{2}}\left(\alpha^{2}-\frac{1}{4}\right)
$$

The coordinate $\chi$ measures the time that it takes a wave to reach a distance $s$ along the string from the free end. Even though the sound speed vanishes at $s=0$, the time for sound waves to come from the free end is finite. Substituting this result into Eq. (11) yields

$$
\frac{\partial^{2} g}{\partial \chi^{2}}+\left(\omega^{2}-\frac{1}{\chi^{2}}\left(\alpha^{2}-\frac{1}{4}\right)\right) g=0
$$

Substituting $g(\chi)=\sqrt{\chi} h(\chi)$ gives Bessel's equation

$$
\chi^{2} \frac{\partial^{2} h}{\partial \chi^{2}}+\chi \frac{\partial h}{\partial \chi}+\left(\chi^{2} \omega^{2}-\alpha^{2}\right) h=0
$$

so we have

$$
g(\chi)=\sqrt{\chi}\left[c_{1} J_{\alpha}(\omega \chi)+c_{2} Y_{\alpha}(\omega \chi)\right]
$$

Because the string has a finite length $L$, the variable $\chi$ varies from $\chi_{1}=0$ to

$$
\chi_{2}=2 \sqrt{\frac{L}{g}(\alpha+1)}
$$

How the string is attached at $L$ determines a boundary condition at $\chi_{2}$. For example, if the string is fixed at $L$, $\chi_{2}$ must be a zero of the function $g(\chi)$. At the free end $\left(\chi_{1}=0\right)$ the displacement of the string must not diverge so $c_{2}=0$. These two boundary conditions determine the spectrum of values for $\omega$.

\section{Power-Law Tension}

Let's assume a more complicated dependence of the force on the distance along the string. Specifically let us take

$$
d \lambda=q s^{\beta} \mu d s=q b s^{\alpha+\beta} d s
$$

So

$$
\lambda(s)=\frac{q b}{\alpha+\beta+1} s^{\alpha+\beta+1}, c_{s}=\sqrt{\frac{q s^{\beta+1}}{\alpha+\beta+1}}
$$

The coordinate $\chi$ is now a bit more complicated

$$
\chi=\frac{2}{1-\beta} \sqrt{\frac{\alpha+\beta+1}{q}} s^{(1-\beta) / 2}+C
$$

If we take $\beta=0$ and $q=g$, we obtain Eq. (14), and if $\beta=1$ we have

$$
\chi=\sqrt{\frac{2+\alpha}{q}} \ln s+C
$$

Specifically if $\beta \geq 1$ it takes a infinite amount of time for a wave to propagate from the free end of the string upward. The free end of the string is a horizon for waves travelling along the string. For $\beta<1$ is the natural to set the arbitrary constant of integration $C$ equal to zero, so that $\chi$ gives the amount of time for a excitation at the 
end of the string to reach a point a distance $s$ away. For $\beta \geq 1, C$ may be defined conveniently by taking the zero point of $\chi$ to be the fixed end of the string $s=L$.

In general we have

$$
a(s)=\left(\frac{b^{2} q}{\alpha+\beta+1}\right)^{1 / 4} s^{(2 \alpha+\beta+1) / 4} .
$$

and

$$
a(\chi)=\left(\frac{b^{2} q}{\alpha+\beta+1}\right)^{1 / 4}\left(\frac{1-\beta}{2} \sqrt{\frac{q}{\alpha+\beta+1}} \chi\right)^{\frac{2 \alpha+\beta+1}{2-2 \beta}}
$$

For $\beta \neq 1$ we have

$$
m_{\mathrm{eff}}^{2}=\frac{1}{\chi^{2}} \frac{(2 \alpha+3 \beta-1)(2 \alpha+\beta+1)}{4(1-\beta)^{2}} .
$$

The effective mass-squared is bounded from below by $-1 /\left(4 \chi^{2}\right)$ just as for Eq. (7) so the solutions developed earlier apply for arbitrary values of $\beta$ but with different boundary conditions if $\beta \geq 1$. If $\beta \geq 1$, the end of the string $(s=0)$ corresponds to $\chi \rightarrow-\infty$, so one gets a continuous spectrum of eigenvalues $\omega$.

For $\beta=1$ we have

$$
m_{\mathrm{eff}}^{2}=q \frac{\alpha+1}{4}
$$

In this special case, the function $g(\omega, \chi)$ does not satisfy Bessel's equation but a simpler version of Eq. (11) with a constant value of $m_{\text {eff }}^{2}$. Here both the energy and the $\chi$-momentum of the modes are conserved and we have

$$
\hat{u}(\chi, t)=e^{-i(k \chi-\omega t)} \hat{a}_{\omega}+e^{i(k \chi-\omega t)} \hat{a}_{\omega}^{\dagger}
$$

with $\omega^{2}=k^{2}+m_{\text {eff. }}^{2}$. Although the picture looks simple in terms of the $\chi$ variable, the reality is a bit more complicated. The string has a finite length over which $\chi$ runs from $-\infty$ to 0 from Eq. (24).

\section{Setting the Tension}

Fixing the tension in the string to follow an arbitrary function is actually less complicated than it might first appear. Because the analysis is concerned with oscillations in only a single direction (to be definite call this the $y$-direction), the string can be forced to lie upon a particular surface $z=z(x)$; thereby, setting the tension. If we resolve the downward acceleration of gravity $(g)$ along the direction of the string, we find that the tension increases along the string as

$$
d \lambda=\mu \cos \theta g d s=\mu g \frac{d z}{d s} d s=\mu g d z
$$

where $\theta$ is the angle that the string makes with the vertical at a particular location. If the mass-density along the string is constant $(\alpha=0)$, we have

$$
\lambda=\mu g z=\frac{q \mu}{\beta+1} s^{\beta+1}
$$

where have used Eq. (22). Two immediate solutions come to mind. First, the trivial solution for $\beta=0$ is simply $z=s$, the string hangs vertically. Second, $\beta=1$ yields

$$
g z=\frac{q}{2} s^{2} .
$$

If one looks at a particle sliding along a curve that satisfies Eq. (32), the potential energy is proportional to the square of the displacement - so the frequency of the oscillatory motion is independent of the amplitude. The curve with this property is called the tautochrone or isochronous. Huygens 12] found that the cycloid satisfies Eq. (32) (also see [13]),

$$
x=\frac{g}{8 q}(\theta-\sin \theta), z=\frac{g}{8 q}(\cos \theta-1) .
$$

For general values of $\alpha$ and $\beta$ the curve must satisfy the following differential equations

$$
\frac{\mathrm{d} z}{\mathrm{~d} s}=\frac{q}{g} s^{\beta}, \frac{\mathrm{d} x}{\mathrm{~d} s}=\sqrt{1-\frac{q^{2}}{g^{2}} s^{2 \beta}} .
$$

The first equation results from combining Eq. (21) and Eq. (31) - the dependence on the mass density vanishes. The second equation results from the Pythagorean theorem.

The solution to these equations is

$$
\begin{aligned}
& z=\frac{s}{(\beta+1)}\left(\frac{q}{g} s^{\beta}\right) \\
& x=s_{2} F_{1}\left(\left[-\frac{1}{2}, \frac{1}{2 \beta}\right],\left[1+\frac{1}{2 \beta}\right],\left(\frac{q}{g} s^{\beta}\right)^{2}\right)
\end{aligned}
$$

where ${ }_{p} F_{q}$ denotes the generalized hypergeometric function. Let's denote this family of parametric curves, hypercycloids. The value $\beta=1$ yields the usual cycloid and the value $\beta=0$, a vertical line.

Fig. 1] depicts the hypercycloids from $\beta=0.5,1,2,3$ and 4 . The curves are symmetric about the $z=0$ line and can be scaled in the $x$ - and $z$-direction simultaneously. This changes the value of $g / q$. However, the two directions cannot be scaled independently. The cycloid $(\beta=1)$ is the critical curve that admits a horizon to string vibrations at $x=z=0$.

If the tension is some more general function $\lambda(s)$, we can use Eq. (30) to determine the necessary curve through the following equations

$$
\frac{\mathrm{d} z}{\mathrm{~d} s}=\frac{1}{\mu g} \frac{\mathrm{d} \lambda}{\mathrm{d} s}, \frac{\mathrm{d} x}{\mathrm{~d} s}=\sqrt{1-\left(\frac{\mathrm{d} z}{\mathrm{~d} s}\right)^{2}}
$$

Because $a^{2}(s)=c_{s}(s) \mu(s)=\sqrt{\lambda(s) \mu(s)}$, Eq. (37) allows us to build a string analogue to a wide variety of conformally flat spacetimes. 


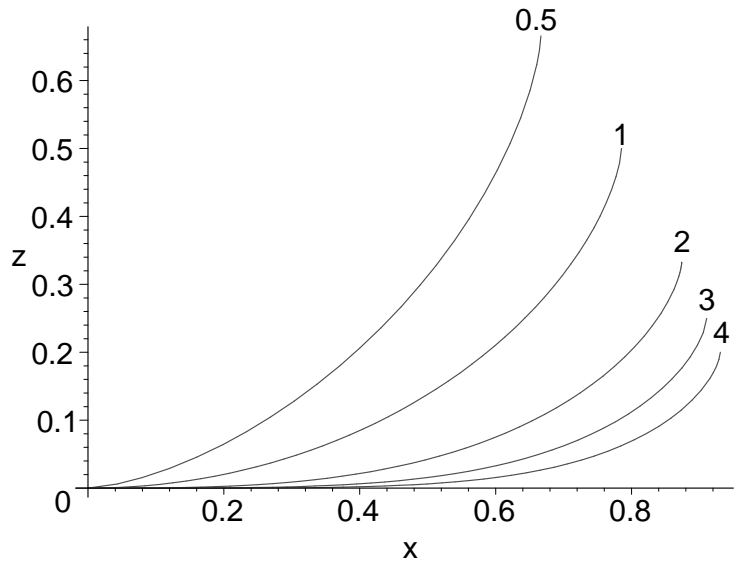

FIG. 1: The cross-section of the confining surfaces, hypercycloids for $\beta=0.5,1$ (the cycloid), 2, 3 and 4 from left to right. The curves are labelled with the value of $\beta$

\section{STRING HORIZONS}

In the previous section, we characterize the mechanics of oscillations of a string with a varying mass density and tension in terms of an effective acoustic metric. It is possible to identify the conformally flat spacetime that describes the motion of waves along a string with spherically symmetric scalar fields in static, spherically symmetric spacetimes such as black holes.

The spacetime of a static, spherically symmetric system may be described using isotropic coordinates [14]

$$
d s^{2}=B^{2}(\bar{r}) d t^{2}-A^{2}(\bar{r}) d \overline{\mathbf{x}}^{2}
$$

where $\bar{r}^{2}=\bar{x}^{2}+\bar{y}^{2}+\bar{z}^{2}$. The spatial portion of this metric is conformally flat.

Let's write the action for a scalar field in this spacetime,

$$
\begin{aligned}
S & =\frac{1}{2} \int d^{4} x \sqrt{-g}\left[\partial_{\mu} \phi \partial^{\mu} \phi\right] \\
& =\frac{1}{2} \int d^{4} x A^{3}(\bar{r}) B(\bar{r})\left[\frac{1}{B^{2}(\bar{r})}\left(\frac{\partial \phi}{\partial t}\right)^{2}-\frac{(\nabla \phi)^{2}}{A^{2}(\bar{r})}\right](40)
\end{aligned}
$$

where we have taken $\hbar=c=1$.

Let's focus on spherically symmetric waves to get

$$
\begin{gathered}
S=\frac{1}{2} \int d t \bar{r}^{2} d \bar{r} d \Omega\left[\frac{A^{3}(\bar{r})}{B(\bar{r})}\left(\frac{\partial \phi}{\partial t}\right)^{2}\right. \\
\left.-A(\bar{r}) B(\bar{r})\left(\frac{\partial \phi}{\partial \bar{r}}\right)^{2}\right]
\end{gathered}
$$

The dynamics of a spherically symmetric scalar field in this spacetime can be mimicked by making the identifications

$$
s=\bar{r}, \mu(s)=r^{2} \frac{A^{3}(\bar{r})}{B(\bar{r})}, \lambda(s)=\bar{r}^{2} A(\bar{r}) B(\bar{r}) .
$$

Using Eq. (37) we have

$$
\frac{\mathrm{d} z}{\mathrm{~d} s}=\frac{1}{\mu g} \frac{\mathrm{d} \lambda}{\mathrm{d} s}=\frac{1}{r g A^{2}(\bar{r})}\left\{2+\frac{\mathrm{d} \ln [A(\bar{r}) B(\bar{r})]}{\mathrm{d} \ln r}\right\}
$$

with units with $G=c=1$. Hence the product $r g$ is dimensionless, as are the functions $A(\bar{r})$ and $B(\bar{r})$. Although this equation may be solved for any spacetime (Eq. (38) ), it is useful to look at the form of this equation in the vicinity of a horizon and compare it with Eq. (34).

For example let's take the spacetime of a charged, nonrotating black hole (the Reissner-Nordström metric) [15, [16, 17], we have [18, 19]

$$
\begin{aligned}
& A(\bar{r})=\left(1+\frac{M}{2 \bar{r}}\right)^{2}-\frac{Q^{2}}{4 \bar{r}^{2}} \\
& B(\bar{r})=\frac{1}{A(\bar{r})}\left[1+\frac{1}{4 r^{2}}\left(Q^{2}-M^{2}\right)\right]
\end{aligned}
$$

where we have taken $G=c=1$.

In this case we have

$$
\frac{\mathrm{d} z}{\mathrm{~d} s}=\frac{32 r^{5}}{g\left(\bar{r}^{2}-\bar{r}_{H}^{2}\right)\left[(2 \bar{r}+M)^{2}+Q^{2}\right]^{2}} .
$$

This expression is actually integrable in terms of elementary functions. However, the corresponding $\mathrm{d} x / \mathrm{d} s$ is not. The horizon is located where $B(r)$ vanishes, that is at

$$
\bar{r}^{2}=\bar{r}_{H}^{2}=\frac{M^{2}-Q^{2}}{4} .
$$

In general we find that $\mathrm{d} z / \mathrm{d} s$ diverges as $\left(\bar{r}-\bar{r}_{H}\right)^{-1}$ when $\bar{r} \rightarrow \bar{r}_{H}$; this is difficult to achieve as $|\mathrm{d} z / \mathrm{d} s| \leq 1$ by the Pythagorean theorem. However, if we look at an extremal Reisser-Nordström metric $\left(M^{2}=Q^{2}\right)$, we have $A(r)=1+M / \bar{r}$ and $B(r)=1 / A(r)$ from Eq. (44) and Eq. 45) so

$$
\lambda(s)=\bar{r}^{2}, \mu(s)=\bar{r}^{2}\left(1+\frac{M}{\bar{r}}\right)^{4}
$$

and

$$
\frac{\mathrm{d} z}{\mathrm{~d} s}=\frac{2 \bar{r}^{3}}{g(\bar{r}+M)^{4}}
$$

The expression $\mathrm{d} z / \mathrm{d} s$ reaches a maximum at $r=3 M$ of $27 /(2 g M)$, so if $M>27 /(2 g)$ we can construct a curve to represent the entire spacetime; otherwise we are restricted to the region with $\mathrm{d} z / \mathrm{d} s \leq 1$.

In practice however, the value of the gravitational acceleration on Earth is so small $\left(\sim 10^{-16} \mathrm{~m}^{-1}\right)$ that $M$ must be greater than $10^{17} \mathrm{~m}$ to emulate the entire extremal Reissner-Nordström spacetime, so terrestrial experiments will necessarily focus on $r \ll M$, the region near the horizon and we have

$$
\frac{\mathrm{d} z}{\mathrm{~d} s} \approx \frac{2 \bar{r}^{3}}{g M^{4}}
$$


This yields the hypercycloid with $\beta=3$ Unfortunately the mass density diverges when $r \rightarrow 0$ as

$$
\mu(s) \approx \frac{M^{4}}{\bar{r}^{2}}
$$

so emulating even the region of this spacetime near the horizon may be difficult. Perhaps other spacetimes that lie at the critical point of having a horizon may also exhibit simple string analogues like Reissner-Nordström.

\section{PROSPECTS}

The theoretical framework outlined here is not complete. Specifically, the Lagrangian, Eq. (11), assumes that the presence of the oscillation does not affect tension in the string. Although recent work [5] indicates that the interesting properties of horizons such as horizon radiation 2] are robust with respect to general assumptions of the dispersion relation at high frequencies, the change in the tension in the string due to the oscillation itself is a form of non-linear back-reaction that is not covered by this analysis. The string may offer an avenue to explore both quantum and classical back-reactions on otherwise static spacetimes. Additionally the dispersion relation for waves on a string is probably more complicated that that described by Eq. (1), so the issue discussed in ref. 5] may also be important.

The dynamics of strings has often presented surprises. A common example is the cracking of a bullwhip [20] that indicates that the speed of the tip of the whip has exceeded that of sound. This letter focuses on the classical and quantum dynamics of freely hanging and supported tapered strings (the bullwhip is a particular case). The acoustics of a waves along the string naturally admits a Lorentzian description by means of an acoustic metric. By varying the tension or the mass-density of the string, the acoustics can emulate the causal structure of variety of spacetimes. This letter has focused on conformally flat spacetimes and spherically symmetric black holes in $(3+1)$ dimensions. It is straightforward theoretically to produce acoustic horizons on strings (a string resting on a concave-up cycloid is a limiting case); perhaps, the experimental realization of such systems will provide new insights into the small-scale structure of spacetime.

\section{Acknowledgments}

The author acknowledges support from NSERC. This work made use of NASA's Astrophysics Data System.
[1] J. D. Bekenstein, Phys. Rev. D7, 2333 (1973).

[2] S. W. Hawking, Commun. Math. Phys. 43, 199 (1975).

[3] W. G. Unruh, Physical Review Letters 46, 1351 (1981).

[4] G. E. Volovik, Universe in a Helium Droplet (Oxford University Press, Oxford, 2003).

[5] W. G. Unruh and R. Schützhold, Phys. Rev. D71, 024028 (2005).

[6] C. Barceló, S. Liberati, and M. Visser, Phys. Rev. A68, 053613 (2003).

[7] R. Schützhold and W. G. Unruh, Physical Review Letters 95, 031301 (2005).

[8] C. Barceló, S. Liberati, S. Sonego, and M. Visser, New Journal of Physics 6, 186 (2004).

[9] M. Cadoni, Classical and Quantum Gravity 22, 409 (2005).

[10] H. Goldstein, Classical Mechanics, 2nd ed. (AddisonWesley, Reading, Massachusetts, 1980).

[11] H. Goldstein, C. Poole, and J. Safko, Classical Mechan- ics, 3rd ed. (Addison-Wesley, San Francisco, 2002).

[12] C. Huygens, Horologium oscillatorium (F. Muguet, Paris, 1673).

[13] H. Melville, in Moby Dick (Oxford, Oxford, 1999), Chap. 96 "The Tryworks", originally published in 1851.

[14] C. Misner, K. S. Thorne, and J. A. Wheeler, Gravitation (W. H. Freeman, San Francisco, 1973).

[15] H. Reissner, Ann. Phys. (Berlin) 59, 106 (1916).

[16] G. Nordström, Proc. K. Ned. Akad. Wetensch 20, 1238 (1918).

[17] H. Weyl, Ann. Phys. (Berlin) 54, 117 (1917).

[18] C. W. Misner and J. A. Wheeler, Annals of Physics 2, 525 (1957).

[19] M. Bailyn and D. Eimerl, Phys. Rev. D5, 1897 (1972).

[20] A. Goriely and T. McMillen, Physical Review Letters 88, 244301 (2002). 